\title{
TELETRABAJO, GÉNERO Y GENTRIFICACIÓN O ELITIZACIÓN* EN LOS ESPACIOS RURALES: NUEVOS USOS Y NUEVOS PROTAGONISTAS. LOS CASOS DE CATALUÑA Y ARDĖCHE (FRANCIA)**
}

\author{
Gemma Cánoves Valiente y Asunción Blanco Romero \\ Universitat Autònoma de Barcelona. Dpto. de Geografía, Fac. de Filosofía \\ y Letras. Edificio B. 08193-Bellaterra (Barcelona) \\ Gemma.canoves@uab.es - Asunción.Blanco@uab.es
}

\begin{abstract}
Resumen: Los espacios rurales están cambiando, tanto por sus usos como por sus pobladores. El artículo muestra estas nuevas dinámicas a través del teletrabajo, la gentrificación o elitización y los motivos de género que impulsan a las mujeres a formar parte de estas nuevas iniciativas para convertirse en teletrabajadoras. El ejercicio comparativo de dos casos como el de Cataluña y el Ardeche en Francia, nos sitúa en realidades diferentes y en dinámicas temporales con una evolución distinta. El material sobre el que se ha trabajado combina los datos estadísticos con las entrevistas en profundidad, imprescindibles para estas investigaciones. El resultado muestra que el teletrabajo es una opción poco consolidada en Cataluña, que las mujeres adoptan esta formula en momentos puntuales y que los espacios rurales están incorporando nuevos pobladores, con claras estrategias de diversificación social y económica.
\end{abstract}

Palabras Clave: Teletrabajo, género, gentrificación, elitización, Cataluña, Ardeche

\begin{abstract}
Rural spaces present a clear change in social composition and uses. The present article shows these new dynamics by gender composition, teleworking and gentrification. The article compares the cases of Catalonia and Ardeche in France, which are in different dynamics and temporal periods. The material research has combined statistics and in-deep interviews. The results show tele-
\end{abstract}

* Recibido: 12-12-2005. Aceptado: 29-5-2006.

** El Consejo de redacción de Geographicalia ha decidido publicar nuevamente en este número de la revista el artículo de Gemma Cánoves y Asunción Blasco, ya que por error se incluyó en el número anterior la primera versión del mismo, sin las correcciones que los evaluadores habian sugerido y las autoras incorporaron al texto. 
work as a non-consolidated option in Catalonia; also exposes that women adopt this formula in punctual moments, and rural spaces have incorporated new population with clear strategies of social and economic diversification

Key words: Telework, gender, gentrification, Catalonia, Ardeche'1.

\section{Introducción}

Los espacios rurales están cambiando, tanto por sus habitantes como por sus usos. Este artículo pone de manifiesto dos cambios recientes que se han detectado en Cataluña y Ardèche en relación a la elitización del espacio rural: el surgimiento del teletrabajo y los motivos de género para adoptar esta nueva forma de trabajo. El artículo se fundamenta en un destacado trabajo de campo (50 entrevistas en profundidad y 50 cuestionarios) realizado durante los años 2000 y 2004 (Blanco, 2005). Las investigaciones en esta temática, por la falta de datos estadísticos, no se pueden apoyar únicamente en datos numéricos y por ello requieren de una metodología más cualitativa, con el tratamiento de la información obtenida a partir de las entrevistas en profundidad.

El artículo se concreta en cuatro apartados y unas conclusiones. En el primero, explicamos las zonas de estudio, sus características y la metodología de investigación. A continuación, nos centramos en la explicación de los procesos de gentrificación que se están produciendo en los espacios rurales. Seguidamente, ponemos de manifiesto la relación entre las nuevas actividades surgidas en las zonas rurales y los procesos de género que hay inmersos. Los dos últimos apartados son, en parte, las conclusiones del artículo, donde se reflexiona en relación a los nuevos usos y usuarios de los espacios rurales, para finalmente referimos a los intensos cambios que están sucediendo en los espacios rurales.

\footnotetext{
1 La denominación de gentrificación es un anglicismo de la palabra correcta y utilizada en inglés Gentrification. La ausencia de una traducción, que responda a un proceso de substitución de población de clase social más baja por la llegada de nuevas poblaciones con mayor poder adquisitivo, se ha estudiado de forma profunda y ha sido aplicado en el análisis de las áreas urbanas. Sin embargo poco se ha referido al mundo rural. La terminología de elitización, utilizada por Luz Marina García (2001) en su artículo "Elitización, propuesta en español para el término gentrificación" analiza el fenómeno y reflexiona sobre la terminología utilizada. En nuestro artículo utilizamos de forma indistinta los dos términos, con el objetivo de llegar a asimilar este fenómeno con la designación de elitización.
} 


\section{Las zonas de estudio}

Las zonas seleccionadas para el estudio responden a lo que se considera dos niveles de desarrollo e implantación del teletrabajo en territorios y entornos socioculturales muy diferentes, como la zona del Ardèche (perteneciente al departamento RhôneAlpes en Francia) y Cataluña (España). Al mismo tiempo, Cataluña y Ardeche pertenecen, junto a Baden-Württemberg y Lombardía, al llamado grupo de los "Cuatro Motores para Europa". Consorcio creado con la finalidad de favorecer la cooperación interregional europea y que tiene como objetivos prioritarios la investigación y la innovación.

Por estos motivos, la región de Ardèche ha sido realmente la zona precursora de este análisis. Ardèche, cuenta con 294.522 habitantes, de los cuales el 48\% es población rural ${ }^{2}$. Su crecimiento natural es nulo, por lo que la actual variación demográfica anual media se produce debido a un saldo migratorio positivo. El 25,7\% de la población cuenta con más de 60 años, lo que indica el alto nivel de envejecimiento de la zona. Es una región eminentemente rural, donde el 90\% de las 339 municipalidades que lo forman son consideradas rurales, y únicamente 37 son zonas urbanas. (Ver tabla 1).

Estas características han hecho que el departamento se convierta en objetivo prioritario de los programas y ayudas de los fondos para el desarrollo de la Unión Europea, factor favorable para el desarrollo de iniciativas interesantes en nuestro campo de estudio.

Las áreas de estudio en las que se ha realizado el trabajo de campo (25 entrevistas en profundidad, a 17 mujeres y 8 hombres) forman un grupo de 14 municipios de diferentes zonas de la región (mapa 1).

Estos municipios muestran cierta diversidad a nivel demográfico, si bien todos

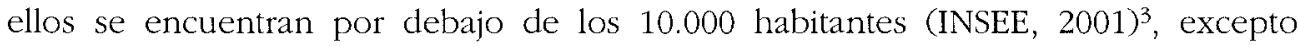
Annonay (con 17.522 habitantes) y Aubenas (11.000 hab.), dos de las tres únicas ciudades que sobrepasan los 10.000 hab. en toda la zona (tabla 2).

En ellos, el sector terciario es reducido, formado principalmente por servicios de consumo, como en Vallon-Pont-d'Arc, ampliamente dedicado al turismo de deportes de aventura. También encontramos el caso de algunos profesionales liberales (abo-

2 Fuente, INSEE: www.insee.fr

3 Datos del Institut de la Statistique et des êtudes Économiques de Francia (INSEE): www.insee.fr/fr/insee_regions/rhone-alpes/zoom/chif_cles/chiffres_cles.htm 
Tábla 1. Rasgos principales de la zona de Ardèche (Rhône-Alpes).

\begin{tabular}{|ll|}
\hline & Datos geográficos \\
\hline Superficie & $5.529 \mathrm{~km}$. \\
Porcentaje de superficie forestada & $57,20 \%$ \\
Ciudades más pobladas & Annonay: 17522 habitantes \\
Número de distritos & Privas, Tournon-sur-Rhône, Largentière \\
Número de regiones (cantón) & 33 \\
Número de municipio & 339 \\
5 Regiones naturales & Montaña, meseta del haut-Vivarais y las Cévennes, \\
& Bas-Vivarais, meseta de Coirons, valle del Rhône. \\
\hline & Demografia \\
\hline Número de habitantes & 294522 hab. (5\% de Rhône-Alpes) \\
Hombres & 140412 \\
Mujeres & 145748 \\
Edad media & 40,3 años \\
Menores de 20 años & $23 \%$ \\
Mayores de 60 años & $26 \%$ \\
İndice de envejecimiento & $2 / 3$ municipios envejecidos \\
Densidad media por km_- & 52 habitantes (122h/ km_ Rhône-Alpes) \\
Concentración población & $70 \%$ población en $2 \%$ del territorio \\
Tasa anual media (Variación 1990-1999) & $+0,33$ \\
Variación gracias al saldo migratorio & $+0,33$ \\
Tamaño medio de las familias & 2,4 \\
\hline & Empleo \\
\hline & 124160 \\
\hline Población activa & 11051 \\
Demandantes de ernpleo (enero 2004) & $10,1 \%$ \\
\hline Tasa de paro & Agricultura \\
\hline & 7090 \\
\hline Número de explotaciones agrícolas & 144330 hectáreas \\
SAU de las explotaciones & $7,40 \%$ \\
Parte del empleo agrícola en el total & \\
\hline
\end{tabular}

Fuente: elaboración propia a partir de los datos del INSEE, de la prefectura de l'Ardèche y otros?.

gados, arquitectos, ingenieros), los cuales han regresado a la zona después de haber llevado a cabo su formación fuera del departamento; o incluso neorrurales, llegados al Ardèche en busca de una mejor calidad de vida.

El caso de Cataluña es el complemento para un estudio comparado. Cataluña se encuentra en un proceso incipiente de desarrollo del teletrabajo y con un escaso nivel

7 Para ampliar información ver:

www ardeche.pref.gouv.fr/departement/PresentationArdeche/geographie.asp

www.insee.fr/fr/region/tabcomp/RGAMTD02.htm

www.cg07.fr $/$ site $/$ site.php?ens $=1 \& r u=3 \& s s \_r u=82 \# a 2_{2}$ 
Mapa 1. Localización de los municipios estudiados en Ardèche (Francia).

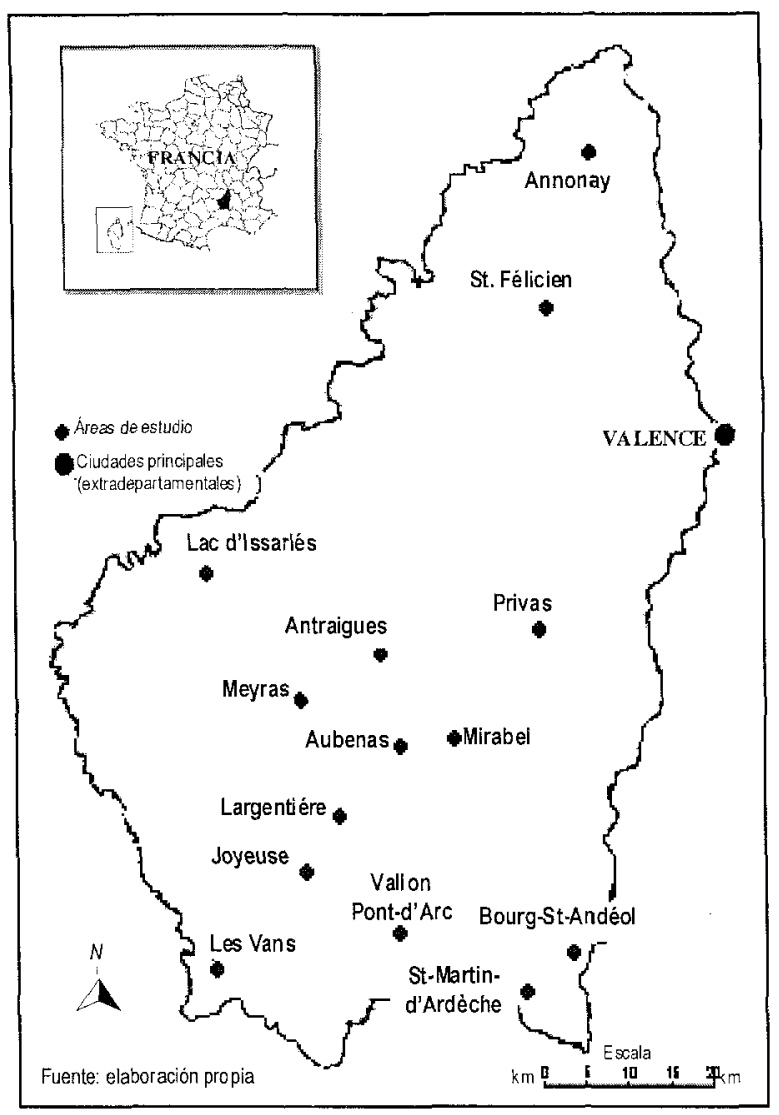

de implantación en el territorio; por ello cuenta con financiación de la Unión Europea para la creación de los llamados telecentros ${ }^{4}$. (Tabla 3 y Mapa 2).

En el caso de Cataluña existe una clara disparidad en el volumen demográfico de los núcleos seleccionados 5 y se constata el reducido carácter rural de la mayoría de las áreas analizadas. Ello es, en parte, consecuencia de la expansión residencial, dada

\footnotetext{
4 Uno de los principal ejemplos es el del programa europeo TeletreBages de la comarca del Bages: www.bages.org/teletrebages/projecte.htm

5 Población de los municipios analizados: en el Bages (Castellbell i el Vilar, 2.904 hab.; Cardona, 5.515 hab.; Mura, 221 hab.; Navàs, 5.580; San Fruitós de Bages, 5.719 hab.), en el Baix Penedès (L'Arboç, 4.008 hab. y El Vendrell, 25.705 hab.) y en el Vallès Oriental (Castellterçol, 2018 hab.; Mollet del Vallès, 46.897 hab. y Montmeló, 8.912 hab.). Idescat
} 
Tabla 2. Datos básicos municipios seleccionados en Ardèche.

\begin{tabular}{|lcccc|}
\hline Municipio & Sup. km. & $\begin{array}{c}\text { \% sup. } \\
\text { departamental }\end{array}$ & Población & Densidad hab/ km. \\
\cline { 2 - 4 } Annonay & 21,2 & 0,38 & 17.522 & 826 \\
Aubenas & 14,32 & 0,26 & 11.000 & 768 \\
Privas & 12,14 & 0,22 & 9.170 & 755 \\
Bourg-Saint-Andéol & 43,74 & 0,79 & 7.768 & 177 \\
Les Vans & 31,09 & 0,56 & 2.664 & 86 \\
Vallon-Pont-d'Arc & 28,62 & 0,52 & 2.027 & 71 \\
Largentiére & 7,22 & 0.13 & 1.942 & 269 \\
Joyeuse & 13,04 & 0,23 & 1.483 & 114 \\
Saint-Félicien & 21,44 & 0,38 & 1.183 & 55 \\
Antraigues & 13,46 & 0,24 & 498 & 37 \\
Meyras & 12,31 & 0,22 & 774 & 63 \\
Saint-Martin-d'Ardèche & 5,53 & 0,10 & 642 & 116 \\
Mirabel & 19,9 & 0,36 & 313 & 16 \\
Le Lac d'Issarlés & 14,35 & 0,26 & 253 & 18 \\
\hline
\end{tabular}

Fuente: elaboración propia a partir de INSEE: www.insee.fr y www.linternaute.con/ville

Tabla 3. Rasgos principales de la zona de Cataluña.

\begin{tabular}{|ll|}
\hline \multicolumn{2}{|c|}{ Datos geográficos } \\
\hline $\begin{array}{l}\text { Superficie } \\
\text { Porcentaje de superficie forestada }\end{array}$ & $32.000 \mathrm{~km}$. \\
Ciudades más pobladas & $43.30 \%$ \\
Número de comarcas & Barcelona: 1.593 .075 habitantes \\
Número de municipio & 41 \\
\hline \multicolumn{2}{|c|}{ Demografia } \\
\hline Número de habitantes (2003) & 646 \\
Hombres & $49,404.000$ hab. \\
Mujeres & $50,6 \%$ \\
Edad media & 40,7 años \\
Menores de 20 años & $19,2 \%$ \\
Mayores de 60 años & $22,1 \%$ \\
Densidad media por km_ & 210 habitantes \\
Crecimiento vegetativo & 1,66 \\
Tamaño medio de las familias & 2 \\
\hline \multicolumn{2}{|c|}{ Empleo } \\
\hline \multicolumn{2}{|c|}{ Agricultura } \\
\hline Deblación activa & 3.050 .000 \\
Tasa de paro & 281.000 \\
\hline \multicolumn{2}{|c|}{$2,5 \%$} \\
\hline Empleo sector agrícola (2001)
\end{tabular}

Fuente: elaboración propia a partir de los datos de IDESCAT:

www.idescat.es/publicacions/caltaleg/pdfdocs/xifresct/xifres_cat04.pdf c INE: www.ine.es 
Tabla 4. Datos básicos municipios seleccionados en Cataluña.

\begin{tabular}{|lcccc|}
\hline Comarca/municipio & Sup. km. & $\begin{array}{c}\text { \% sup. } \\
\text { comarcal }\end{array}$ & Población & Densidad hab/ km. \\
El Bages & & & & \\
Castellbell i el Vilar & 28,80 & $2,24 \%$ & 2.904 & 101 \\
Cardoná & 66,38 & $5,15 \%$ & 5.515 & 83 \\
Mura & 53,39 & $4,15 \%$ & 221 & 4 \\
Navàs & 81,70 & $6,35 \%$ & 5.580 & 68 \\
Sant Fruitós del Bages & 22,40 & $1,74 \%$ & 5.719 & 255 \\
Baix Penedès & & & & \\
L'Arboç & 14 & $4,73 \%$ & 4.008 & 71 \\
Albinyana & 19,56 & $6,62 \%$ & 1.380 & \\
Vallès Oriental & & & & 63 \\
Castellterçol & 32 & $3,75 \%$ & 2.018 & 170 \\
Santa Maria de Martorelles & 4 & $0,47 \%$ & 678 & 64 \\
Vallgorguiná & 22 & $2,58 \%$ & 1.413 & \\
\hline
\end{tabular}

Fuente: ełaboración propia a partir de Idescat: www.idescat.es y Conarcalia: www.comarcalia.com

su proximidad al área metropolitana de Barcelona. Por su menor ruralidad -la casi totalidad de los municipios supera los 2.000 habitantes- cabría esperar un mayor desarrollo del teletrabajo y en cambio ha sido todo lo contrario. La dificultad en encontrar teletrabajadores/as en áreas rurales catalanas se ha visto intensificada si tenemos en cuenta que, a pesar de que el $68 \%$ del total de municipios de Cataluña son menores de 2.000 habitantes, éstos acogen únicamente a un $6 \%$ de la población total catalana, es decir, 383.000 personas $^{6}$ (Tabla 4.).

Por ello, en el caso de Cataluña fue especialmente complicado realizar las 25 entrevistas ( 15 mujeres y 10 hombres) las cuales se distribuyeron en 9 municipios de 3 comarcas concretas: el Bages, el Baix Penedès y el Vallés Oriental (Mapa 2).

La realidad y dinámica de estos dos espacios rurales, nos lleva a plantearnos los procesos que se están produciendo en las zonas rurales. Por un lado, existe un movimiento de población de las grandes ciudades hacia núcleos con menores dimensiones pero con infraestructuras básicas; como es el caso de la comarca del Bages en Cataluña y el Ardeche en Francia. Por otro lado, asistimos a la implantación de actividades en el espacio rural alejadas del sector tradicional primario y que se pueden ubicar en cualquier espacio, debido al desarrollo de las nuevas tecnologías, como es el caso del teletrabajo y finalmente; vemos que son mayoritariamente las mujeres las que adoptan estas nuevas formas de trabajo a distancia, con el objetivo de poder compatibilizar, o al menos flexibilizar, sus jornadas laborales y domésticas. Estas múl-

"Fuente: Idescat, INE, Censo de población 2001. 
Mapa 2. Localización de los municipios estudiados en Cataluña.

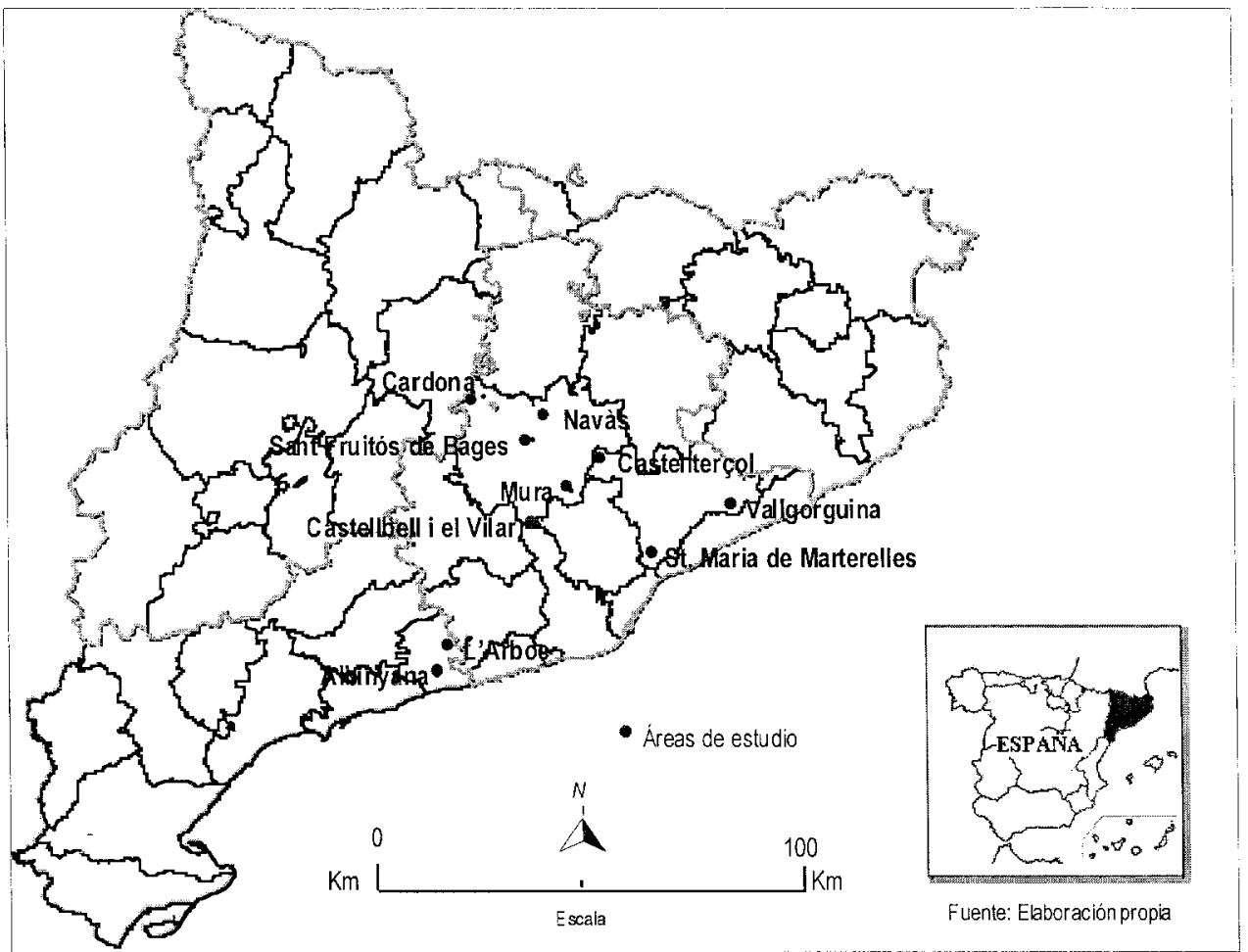

tiples dinámicas, están modificando pautas sociales, culturales y económicas de estos espacios y consideramos que vislumbran un proceso similar de gentrificación o elitización, como el que se ha producido en grandes núcleos urbanos.

\section{Gentrificación o elitización en los espacios rurales}

Los recientes cambios en los espacios rurales nos llevan a constatar un incipiente proceso de elitización. Los procesos de gentrificación han sido estudiados y constatados en entornos urbanos (Butler, 1997). Es conocido el proceso de expulsión de población de menor poder adquisitivo en barrios que se mejoran y que vienen a poblarse por clases sociales con mayor poder adquisitivo (Butler y Savage, 1995). En el reciente artículo de Phillips (2004) se cuestiona y reflexiona sobre los procesos de 
gentrificación en el espacio rural de Inglaterra y en concreto en Nord Norfolk. Este ejercicio comparativo nos da pie a reflexionar si en nuestro país, con fuertes cambios sociales y económicos en los espacios rurales, se está produciendo esta tendencia. A nuestro entender, sí que estamos asistiendo en los últimos quince años a un proceso de elitización de los espacios rurales, tanto desde el ámbito económico, como social y cultural; más todavía si entendemos la gentrificación como un cambio o suplantación económica, poblacional y cultural de personas y modos de vida en los espacios rurales.

La noción de gentrificación implica la renovación de propiedades residenciales, y ello se acompaña de un cambio en la composición social. (Cloke, et al. 1998, Phillips, 1993, 2004). Ejemplos de este proceso se están dando en lugares como el Valle de Arán, o la Cerdanya en Cataluña, zonas donde la economía ha pasado, en los últimos 30 años, de basarse en las actividades primarias a actividades terciarias, centradas en el turismo de esquí y la segunda residencia. El fenómeno ha comportado un incremento sustancial de la segunda residencia, una reconversión de la agricultura hacia la actividad terciaria, y una clara sustitución de la población local por población procedente de las ciudades con un alto poder adquisitivo.

La principal consecuencia es la expulsión de la población joven y local, que ve imposible pagar los precios de las viviendas, debido al fuerte incremento experimentado. A la vez, se sienten invadidos en momentos puntuales y ven sus necesidades mediatizadas por los nuevos llegados. Este proceso también se da en pequeñas poblaciones que, por la proximidad a ciudades importantes y mucho más caras en los costes de la vivienda, están viendo sustituidas sus poblaciones con nuevos residentes y nuevas costumbres; o en pueblos que se ven colonizados, primero en verano y luego todo el año, por nuevos llegados, nuevos residentes, o personas jubiladas, y más recientemente por la llegada de inmigrantes, que atraídos por el dinamismo económico del turismo y la construcción van a localizarse en el medio rural, transformando la estructura social, las costumbres y las dinámicas de estas pequeñas poblaciones.

Los resultados son múltiples y variados, desde una difícil integración, a una relación entre la comunidad local y los nuevos llegados más o menos tranquila, o a veces la inevitable sensación de invasión producida tanto por los turistas, como por los veraneantes o los habitantes pendulares.

El primero que utiliza el termino gentrificación es Glass (1964) y se refiere a renovar o rehabilitar las propiedades en un área, lo que lleva asociado un cambio en la composición social. Este mismo proceso se puede aplicar a los espacios rurales, cada vez más solicitados para usos residenciales, de primera o segunda residencia. Las casas de campo han sido puestas en valor para un uso residencial temporal (segunda residencia) lejos de su uso habitual agrícola, y tras ello han venido unos nuevos pobladores, generalmente de las ciudades, a reutilizar este nuevo espacio con nue- 
vos usos y nuevos valores. Sin embargo, la teoría de la gentrificación asociada a los espacios urbanos o la rentabilidad económica de esta inversión, renovación- rehabilitación, ha estado asociada al incremento patrimonial y de rentas que supone este nuevo uso. No obstante, se ha prestado poca atención al cambio en la composición social que supone la elitización. Más si lo situamos en los espacios rurales, donde la expresión de la elitización es más notable en las nuevas composiciones sociales (inmigrantes y nuevos llegados de la ciudad), que en la generación de rentas de capital (Paniagua, 2002).

Cloke y Little, (1990, p.164) hablan de gentrificación como un dictado del movimiento poblacional de clase hacia áreas rurales accesibles, en donde existe una inmigración de residentes de clase media a costa de las clases bajas. Los primeros estudios de Phillips, (1993, 2002) constataban las rápidas ganancias que se pueden obtener comprando y vendiendo casas en el campo, y la existencia de un número importante de residentes rurales obteniendo substanciosas rentas de este proceso.

En España esta situación se está dando con la recuperación de casas en espacios rurales, que se ponen en venta una vez reconstruidas y rehabilitadas. Los nuevos habitantes son de diferente clase social que sus originales propietarios. La venta se ha producido por cambio o abandono de la actividad agraria, jubilación de sus propietarios, cambio de domicilio, o simplemente para obtener una renta de aquel patrimonio que se va a convertir en un uso residencial. En este proceso de reutilización y revalorización de los espacios rurales y de las casas, existe paralelo e implícito un proceso de postproductivismo del espacio rural. Este espacio esta siendo reutilizado por nuevos usos alejados de los originales usos agrarios y nuevos pobladores que buscan en las poblaciones rurales, mejor calidad de vida y viviendas más económicas.

Ya se ha comentado por múltiples estudios que el espacio rural no es sólo un espacio productivo; es, cada vez más, un espacio multifuncional y multidemandado para nuevos usos (Cànoves, et al. 2006): un uso recreativo, turístico, residencial, paisajistico, productivo, conservacionista, de implantación de actividades industriales y de servicios; y estos nuevos usos lo disputan, revalorizan y en algunos casos lo dinamizan. Pero esta dinamización implica nuevos pobladores con nuevas demandas, nuevos valores económicos y sociales, y nuevos capitales. Siguiendo la puntualización de Wilson (2001), que defiende que el término postproductivismo tiene que ser interpretado en un amplio abanico, consideramos indispensable poner el énfasis en la desvalorización de la tierra y de las construcciones en relación a los capitales agrícolas y su revalorización con respecto a nuevos capitales y usos (Evans, et al. 2002; Kneale, et al. 1992; Murdoch y Marsden,1994).

Asimismo, la elitización rural ha de ser vista como una forma de revalorización de recursos y espacios que han pasado a ser marginales e improductivos para el capital agrario. La reconversión de los establos, cuadras, graneros o pajares en usos bien dis- 
tintos para los que inicialmente se habían construido (como casas de segunda residencia, restaurantes, tiendas, o alojamientos rurales), es un claro ejemplo del proceso de revalorización y reutilización de estos espacios. Pero en el espacio rural no sólo se han reconvertido los espacios con un claro uso agrícola y las casas rurales, sino también otras construcciones, como las iglesias, los colegios, las almazaras, molinos harineros o minas y canteras. Nuevos usos para viejas y tradicionales actividades, y con ellos nuevos pobladores y nuevas rentas, que ya no provienen principalmente de la agricultura sino del sector de los servicios. Pero este proceso exige una nueva dotación de servicios e infraestructuras y en este sentido el teletrabajo, o trabajo a distancia, es una expresión más de la deslocalización, postproductivismo y elitización de estos espacios rurales.

\section{Género y teletrabajo}

Ante las nuevas revalorizaciones del medio rural y un nuevo uso de este espacio, -si las condiciones de infraestructura lo permiten-, un efecto es la relocalización del trabajo, allá donde el trabajador prefiera o pueda. El análisis detallado de lo que ha sucedido en el caso de las personas que han decidido teletrabajar en los espacios rurales de Cataluña y Ardeche, muestra un claro componente de género. (Mokhtarian et al., 1996; Blanco, 2005).

A pesar de los esfuerzos realizados desde las administraciones y sobre todo desde la UE, el teletrabajo no es la fórmula mágica para resolver los problemas del desempleo; sin embargo, hay que reconocerle la flexibilidad que ofrece. Aunque su papel pueda ser débil como fuerza generadora de actividades o de nuevos puestos de trabajo, su incidencia sobre el empleo de determinados grupos sociales y sobre todo para la mujer, merece una atención específica desde un enfoque de género.

La pregunta clave es epor qué las mujeres tienden a adoptar la opción del teletrabajo? La respuesta es sencilla si tenemos en cuenta que las mujeres se sitúan en aquelos puestos de trabajo que más fácilmente les permitan compatibilizar las tareas productivas y reproductivas. (Cánoves y Villarino, 2000).

La tabla muestra el tipo de trabajo por sectores y quién lo realiza mayoritariamente. El análisis del colectivo de mujeres teletrabajadoras, llevado a cabo en el estudio, nos indica las ventajas e inconvenientes expresadas por las mujeres respecto al teletrabajo, tanto en Cataluña como en Ardeche. Las principales motivaciones que llevan a las mujeres a considerar el teletrabajo como alternativa real, se basan en la necesidad de compatibilizar la vida personal y profesional. Evidentemente, el tipo de trabajo realizado a distancia también es condicionante y los casos encontrados nos permiten afirmar que a mayor nivel profesional, mayor nivel de autonomía. 
Tabla 5. Implicaciones en el teletrabajo por sectores (\% según sexo).

\begin{tabular}{|ll}
\hline Secretariado, administración, Introducción de datos & $100 \%$ mujeres \\
consultoria y proyectos & $85 \%$ hombres \\
educación y formación & $60 \%$ mujeres \\
ventas y marketing & $65 \%$ hombres \\
servicios financieros & $98 \%$ hombres \\
investigación y periodismo & $65 \%$ mujeres \\
traducción y grafismo & $65 \%$ mujeres
\end{tabular}

Fuente: Blanco, 2005. Actualización a partir de Huws, 1996.

Tabla 6. Motivaciones expresadas por las teletrabajadoras.

\begin{tabular}{|lc|}
\hline Motivación $^{*}$ & \% de teletrabajdores $^{* *}$ \\
\hline Flexibilidad de horarios & $41,3 \%$ \\
Compatibilizar esfera profesional y familiar & $40 \%$ \\
Reducir los desplazamientos & $33,3 \%$ \\
Insatisfacción en el trabajo tradicional & $12,8 \%$ \\
Mejorar la calidad de vida & $4,6 \%$ \\
Crear un proyecto de autoempleo & $3,3 \%$ \\
\hline
\end{tabular}

Fuente: elaboración propia a partir de los cuestionarios realizados

* Existen otras motivaciones con \% poco significativo.

** Los porcentajes superan el $100 \%$ ya que hay respuestas que tienen en consideración más de una motivación.

A grandes rasgos podríamos señalar la existencia de dos colectivos de teletrabajadoras (Blanco, 2005). Las que tienen un nivel de formación medio alto y que venden los servicios de forma independiente en sectores punteros, como son las informáticas, las diseñadoras gráficas, las periodistas, etc. Y un segundo nivel formado por las teletrabajadoras que realizan trabajos a la "pieza" o "unidad" para diversos clientes y empresas. En esta situación se encuentran las traductoras, las telesecretarias, o aquellas mujeres que realizan trabajos de oficina, contabilidad, gestión de agendas, etc. A la vista de este amplio abanico de actividades, se constata que los factores que afectan a la decisión de teletrabajar no pueden deslindarse del género, pero tampoco de los aspectos sociales y del nivel de formación.

La tradicional dinámica de la doble jornada laboral de las mujeres se expresa en diferentes estrategias, bien por la reducción de la jornada, bien por el trabajo a tiempo parcial, bien por la "supuesta" flexibilidad del teletrabajo.

Los tradicionales estudios de género (Tremosa, 1986; Escario, \& Alberdi, 1987; Van der Veker \& Hernández, 1989; De Cloet, 1995; Phizacklea, 1995; Baylina, 1996) han 
prestado atención al trabajo a domicilio como un medio para compatibilizar la esfera productiva y reproductiva, pero pocos han analizado las consecuencias del teletrabajo para las mujeres en la unidad familiar, (De Cloet, 2000; De Luís \& Martínez, et al. 2004) y menos aún las consecuencias del teletrabajo en la esfera doméstica (Habid \& Conford, 2002). Una clara muestra de ello es que, para una parte importante de teletrabajadoras, madres de familia, el teletrabajo es iniciado, planificado y vivido como una etapa; que posteriormente abandonarán volviendo a su trabajo tradicional, con un horario prefijado. En esta etapa, el teletrabajo les ha permitido permanecer en casa al cuidado de los hijos pequeños. Para otro grupo de mujeres, el teletrabajo es una alternativa, más o menos aceptable, ante una situación de ruptura en su vida profesional que les permite reciclarse y reincorporarse posteriormente al mundo laboral "estándar" (Blanco, 2005).

Las principales quejas de las mujeres teletrabajadoras se centran en la "consabida" dificultad para separar la esfera doméstica de la profesional, los horarios de trabajo y el aislamiento laboral del teletrabajador. El estudio de Felstead y Jewson (2000) indica que numerosas mujeres todavía hoy en día se encuentran con la realidad de asumir una gran parte de los trabajos domésticos. Si bien ello se produce en cualquier situación laboral, el teletrabajo puede contribuir a acentuar el desequilibrio, dado que se realiza en el mismo espacio, la casa, donde se llevan a cabo las tareas domésticas. Es por ello que las teletrabajadoras argumentan la necesidad de cumplir un horario laboral y separar muy bien las tareas laborales y las domésticas.

El teletrabajo parece constituir una alternativa para las mujeres de combinar de manera más confortable la posibilidad de trabajar y tener una vida profesional activa en la esfera doméstico familiar; pero constituye únicamente una solución parcial al verdadero problema de las mujeres en la división en dos esferas diferenciadas: la profesional y la reproductiva. Así, existe el riesgo de que el trabajo en el ámbito del hogar pueda reforzar su rol doméstico, limitar las posibilidades de proyección personal y no resolver los problemas de atención a los hijos. Por ello, la necesidad de separar de forma eficaz espacio y tiempo de trabajo, del dedicado al hogar y la familia, crea en muchos casos la necesidad de buscar las mismas soluciones que adoptan las mujeres que trabajan en el mercado laboral estándar. Sin embargo, Dumas (1985) argumenta que el teietrabajo puede constituir una etapa en los ciclos vitales de las mujeres. En diferentes etapas de la vida (ciclos familiares, de salud, o ciclos laborales) las mujeres muestran un comportamiento diferente en relación al teletrabajo y éste puede constituir una solución temporal, para alcanzar un mejor nivel laboral, permanecer en espera de mejores ofertas, o simplemente salvar el bache familiar que supone una enfermedad o la maternidad.

Es por ello que, para una parte importante de las teletrabajadoras, madres de familia (el 38\% de las entrevistas realizadas), el teletrabajo es iniciado, planificado y vivido 
como una etapa en la que permanecerán o no en función de los resultados y de la experiencia. No obstante, un punto importante para no regresar a un trabajo estándar es la flexibilidad de horarios, la experiencia de ser autónomo y la mayor facilidad para combinar la vida profesional y la personal, sobre todo en los momentos en que los hijos son pequeños. Como muestra el gráfico en la región de Ardèche, donde la implantación del teletrabajo está más consolidada, la mayoría de las entrevistadas muestra su satisfacción ante las posibilidades que este tipo de organización le ofrece. A pesar de todo, siempre existe para las teletrabajadoras el riesgo de conflicto entre el empleo y la familia, principalmente en las primeras etapas de implantación, como muestran los datos en el caso de Cataluña.

Festead y Jewson (2000) señalan que el acceso al tiempo y al espacio en la casa puede ser el objeto de negociaciones y ajustes más difíciles y reiterados para las mujeres. Estas dificuitades, para hacer entender a la familia que ellas trabajan en casa y que no pueden ocuparse de los quehaceres domésticos en su tiempo de trabajo, refleja una vez más la desigualdad en el reparto de las tareas domésticas entre hombres y mujeres.

Otra motivación que expresan las mujeres, que teletrabajan, tanto en Cataluña como en Ardèche, es la dificultad que encuentran en su ámbito laboral para soportar la presión ejercida por los superiores, tener que dar cuentas a un superior y consta-

\section{Gráfico 1. Valoración conciliación trabajo-familia según área de estudio.}

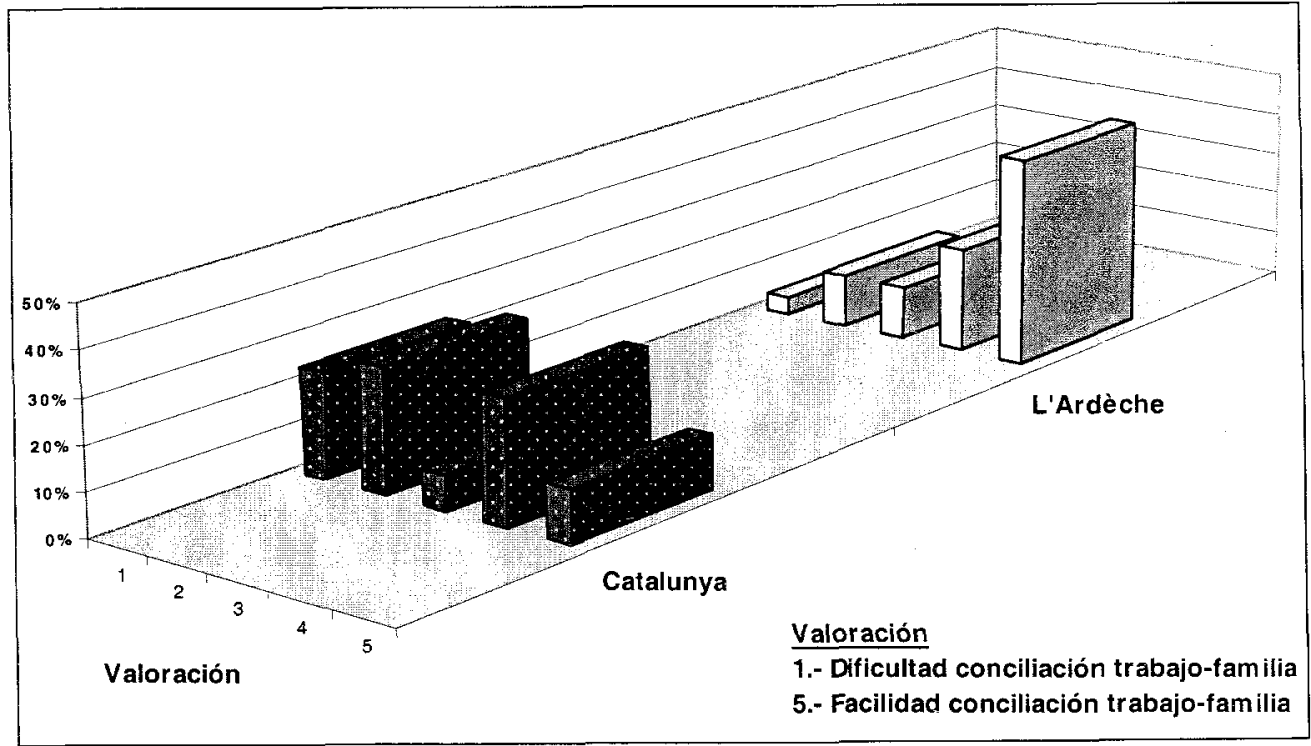

Fuente: Elaboración propia a partir de cucstionarios realizados. 
tar lo restringido de sus oportunidades para desarrollar su carrera profesional de forma autónoma. Así, las facetas positivas que argumentan las mujeres y en general el colectivo de teletrabajadores, se apoyan en las ventajas de la autonomía del trabajo en sí mismo: ser el propio jefe, la posibilidad de autogestionar el tiempo y el ritmo de trabajo, ser independiente y controlar el proceso del producto del inicio al final, reducir el tiempo de los desplazamientos y el estrés del horario laboral y poder mantener una atención continuada a los miembros de la familia y sobre todo a los hijos pequeños.

Por tanto, el teletrabajo no es tan sólo una alternativa para las mujeres con cargas familiares, sino que también puede representar una oportunidad profesional más manejable para mujeres que prefieren controlar ellas mismas su actividad laboral. Es por tanto una opción que permite ventajas para las mujeres, ya que gestionan su tiempo, el tipo y el ritmo del trabajo, son autónomas y no dependen de un jefe, reducen el tiempo y el coste de los desplazamientos y en los momentos en que los hijos son pequeños pueden dedicarles más tiempo.

A pesar de esta visión relativamente optimista, la mayor parte de las teletrabajadoras expresan la inseguridad que supone su decisión, la novedad de su situación, los riesgos económicos que el trabajo autónomo comporta, la reducida protección social y laboral, la falta de reconocimiento social y la invisibilidad de su actividad, que en algunos casos puede suponer problemas de aislamiento.

\section{Conclusiones: nuevos protagonistas y nuevos usos de los espacios rurales}

Los espacios rurales están cambiando, tanto por sus pobladores como por sus usos. Estos cambios se reflejan en las nuevas actividades y percepciones del espacio rural, pero se plasman claramente en las nuevas composiciones sociales que se reflejan en los pueblos pequeños y medianos. Esta transformación se refleja en un proceso de elitización de las sociedades rurales, en nuevos usos de los espacios rurales, en la multifuncionalidad de los territorios y en las nuevas composiciones sociales de estos espacios. El teletrabajo es un reflejo más de esta nueva perspectiva de uso del espacio y de los nuevos pobladores rurales. La descomposición de las estructuras sociales tradicionales ya lleva un largo tiempo produciéndose en el ámbito de Cataluña y de la Unión Europea en general. Este proceso se inició con la transformación de las segundas residencias en primeras y de los residentes de fin de semana en habituales. A continuación, se dio la desvinculación de las nuevas actividades rurales de la tradicional actividad primaria y más recientemente la atracción de los inmigrantes extracomunitarios por los nuevos puestos de trabajo o por las posibilidades 
de establecerse como empresarios. (Lardiés, 1999). Estos cambios están comportando una recomposición social y una diversificación económica, en parte motor del desarrollo local endógeno que experimentan las localidades rurales bien comunicadas y con elementos favorecedores del dinamismo.

Es evidente que no todos los territorios rurales presentan las mismas oportunidades y que no basta con una iniciativa local, o con la implantación de una actividad atractiva; por el contrario, los nuevos emprendedores, las nuevas oportunidades de deslocalización o reubicación de las personas emprendedoras y de las empresas, requieren la existencia de un tejido social amplio, una óptima red de nuevas tecnologías de comunicación y unas infraestructuras viarias adecuadas que permitan la conexión rápida con los centros de decisión.

Una de las alternativas para zonas que no reúnen estas condiciones ha sido la implantación de los telecentros, creados para dinamizar y aplicar la sinergia de ayuda y apoyo a los nuevos emprendedores, principalmente teletrabajadores, facilitándoles la utilización de medios tecnológicos y servicios compartidos a los que, de otra forma, difícilmente podrían acceder.

Si bien estas iniciativas por parte de las administraciones son por ahora puntuales y no permiten garantizar un futuro claro para los espacios rurales, constituyen un buen ejemplo de las nuevas dinámicas puestas en marcha.

En definitiva, el análisis de la realidad de paises que han pasado ya por este proceso, como Francia, Canadá o Gran Bretaña, muestra que la llegada al espacio rural de nuevos pobladores, no originarios del lugar, es un hecho constatado que responde a esta nueva percepción de los espacios rurales. Percepción creada gracias a las mejoras en sus infraestructuras, a una ampliación en la dotación de servicios y equipamientos colectivos, factores de atracción que favorecen la tendencia a equilibrar los niveles de los territorios y a la mejora, en algunos casos, de la calidad de vida en estos nuevos espacios rurales. En este contexto, el teletrabajo y los telecentros no son una alternativa única, pero pueden contribuir a este nuevo repoblamiento de los espacios rurales, imprescindible para conseguir un mejor equilibrio territorial. El estudio realizado en Cataluña y Ardèche pone de manifiesto que las dos zonas responden a dos niveles de desarrollo e implantación del teletrabajo en base a territorios y entornos socioculturales muy diferentes. Mientras en Cataluña el teletrabajo es incipiente y mucho más elemental en sus actividades, en Ardeche es una opción de las mujeres que teletrabajan como una etapa de su proyecto profesional y personal. Es evidente que las condiciones sociales y económicas de ambos lugares son diferentes, pero la particularidad es el momento temporal, la dinámica de la sociedad local y las iniciativas de cada lugar. 


\section{Bibliografía}

Baylina, M. (1996). Trabajo industrial a domicilio, género y contexto regional en la Espana Rural. Tesis Doctoral. Departamento de Geografía. Universitat Autònoma de Barcelona. $378 \mathrm{pp}$.

Blanco, A. (2005). Teletrabajo, género y territorio. Una comparación entre Cataluña, Ardècbe y Québec. Tesis doctoral. Departamento de Geografía. Universitat Autònoma de Barcelona. 478 pp

Butler, T y Savage,M (eds) (1996). Social change and the Middle Classes, UCL, Pres. London. UK. 388 pp.

Butler, $T$ (1997). Gentrification and the Middle Classes. Aldershot. UK Ashgate. $196 \mathrm{pp}$.

Cánoves, G. y Villarino, M. (2000). "Turismo en Espacio rural en España: Actrices e imaginario colectivo". Documents d'Anàlisi Geográfica, 37, pp. 51-77.

Cánoves, G, Villarino, M y Herrera, L. (2006). "Políticas públicas, sostenibilidad y turismo rural en España: difícil equilibrio". Boletin de la Asociación de Geógrafos Españoles. Vol. 41, pp.183-198

Cloke, P. y Little, J. (1990). The rural state? University Press. Oxford, UK. $287 \mathrm{pp}$.

Cloke, P., Phillips, M. y Thrift, N. (1998). "Class, colonization and lifestyle strategies in Gower", en Boyle, P. y Halfacree, K. (eds). Migration Into Rural Areas. Chichester, UK: Wiley.pp.166-185

De Cloet, CH. (1995). "El centro de trabajo telemático: una opción para fomentar el empleo femenino rural", en Cànoves, G. (coord) (1995). La mujer mural. El Campo, $\mathrm{n}^{\circ}$ 133. Servicio de estudios BBV. pp. 295 313 .

De Cloet, CH. (2000). "La revolución telemática: nuevos modelos en el trabajo y la vida de la familia.", en Banús, E. (ed.). El espacio social femenino-Women's social space. Vol. 2. Serie Studia Europa Navarensis. Newbook Edic.

De Luis, P. \& Martínez, A. \& Pérez, M. \& Vela, M.J. (2004). "El enfoque de género aplicado a la relación teletrabajo-medioambiente". IX Jornadas de Economía crítica. Madrid. Marzo 2004. www.ucm.es/info/ ec/jec9/index.htm (Consulta: 11.11.2005).

Dumas, M.C. (1985). Le travail informatisé à domicile et ses implications pour les femmes: une enquête exploratoire auprès de travailleuses à domicile. Mémoire de mâ̂trise. Université du Québec. Montréal.

Escario, P. \& Alberdi, I. (1987). El impacto de las Nuevas Tecnologías en la Formación y el Trabajo de las Mujeres. Serie Estudios, $n^{\circ} 6$. Instituro de la Mujer. Ministerio de cultura. Madrid. $134 \mathrm{pp}$.

Evans, N., Morris, C. y Winter, M. (2002). "Conceptualizing agriculture: a critique of postproductivism as a new orthodoxy". Progress in Human Geography, $\mathrm{n}^{\circ} 26$, pp. 313-332.

Felstead, A Y Jewson, N. (2000). In Home, at Work. Towards an Understanding of Homeworking. Routledge. Londres. 196 pp.

García Herrera, Luz Marina. (2001) "Elitización: una propuesta en español para el término gentrificación" Biblio $3 \mathrm{~W}$ Revista Bibliografica de Geografia $y$ Ciencias Sociales, Universidad de Barcelona, Vol. VI $n^{\circ} 332$

Glass, R. (1964). London: Aspects of change. McGibbon and Kee. London, UK. 343 pp.

Huws, U. (1996). Teleworking and gender. Institute for employment studies. Brighton. UK. 78 pp. 
Kneale,K., Lowe,P. y Marsden,T. (1992). "The conversion of agricultural buildings: an analysis of variable pressures and regulations towards the post-productivist countryside". ESRC countryside Change Initiative. Working paper $n^{\circ} 29$. University of Newcastle.

Lardiés, R. (1999.) Turismo e inmigrantes extranjeros de origen comunitario: $E l$ desarrollo de empresas turisticas en el litoral catalán. Geographicalia, $\mathrm{n}^{0} 37$, pp. 115.

Mokhtarian, P.L. \& Bagley, M.N., Hulse, L. Salomon, I. (1996). The influence of gender and occupation on individual perceptions of telecommuting. Cap. 37. pp. 690711. Second National Conference Women's Travel Issues. Baltimore. Office of Highway Policy Information. www.fhwa.dot.gov/ohim/womens/chap3 7.pdf

Paniagua, A. (2002). "Urban-rural migration, tourism entrepreneurs and rural restructuring in Spain." Tourism Geograpbies. Vol 4, $\mathrm{n}^{\circ} 4$, pp. 349-371.

Phillips, M. (1993). "Rural Gentrification and the process of class colonization". Joumal of Rural Studies, $n^{\circ}$ 9, pp. 123-140.
Phillips, M. (2002) "The production, symbolization and socialization of gentrification: impressions from two Berkshire villages." Transactions of the Institute of British Geographers, no 27, pp. 282-308.

Phillips, M. (2004). "Other geographies of gentrification". Progress in Human geograpby, vol. 28, $\mathrm{n}^{\circ} 1$, pp. 5-30.

Phizacklea, A. \& Wolkowitz, C. (1995). Homeworking women. Gender, racism and class at work. SAGE Publ. London. $152 \mathrm{pp}$.

Murdorch, J. y Marsden, T. (1994). Reconstituting Rurality. London: UCL Press. 256 pp.

Tremosa, L. (1986). La mujer ante el desafio tecnológico. Icaria. Barcelona. 78pp.

Van Der Veker, M. \& Hernández, I. (1989). Mujeres, tecnologia $y$ desarrollo. Serie Estudios, $\mathrm{n}^{\circ}$ 23. Ministerio de Asuntos sociales. Instituto de la Mujer. Madrid. 175 pp.

Wilson, G. (2001). "From productivism to postproductivism... and back again? Exploring the (un) changed natural and mental landscapes of European agriculture". Transactions of the Institute of British Geographers, n ${ }^{\circ} 26$, pp. 77-102. 